\title{
Effective field theory for warm QCD
}

\author{
Sourendu Gupta* and Rishi Sharma ${ }^{\dagger}$ \\ Department of Theoretical Physics, Tata Institute of Fundamental Research, \\ Homi Bhabha Road, Mumbai 400005, India
}

(Received 10 November 2017; published 27 February 2018)

\begin{abstract}
Using only global symmetries of QCD, we set up an effective model of quarks at finite temperature near the crossover, including all possible terms up to dimension-6. We first treat this in mean field theory. Then we investigate low-energy fluctuations around it up to one-loop order in fermions below the crossover. Static correlation functions of pions and the crossover temperature, both measured on the lattice, completely suffice to fix all parameters of the theory. We examine predictions of this theory, including those for thermodynamic quantities. The results are encouraging.
\end{abstract}

DOI: $10.1103 /$ PhysRevD.97.036025

\section{INTRODUCTION}

Effective field theories (EFTs) are good ways of organizing the computation of low-energy or long-distance effects in a quantum field theory. QCD at very low temperature, $T$, seems to be well described by an EFT which describes the dynamics of pions [1,2]. At very high temperature it seems to be possible to understand longdistance phenomena qualitatively in an effective weakcoupling expansion [3]. These rely on a separation of scales $T \gg g T \gg g^{2} T$, where $g$ is the gauge coupling at momentum scale of order $T$. The EFT at each different momentum scale is obtained by integrating over momenta larger than those required at this scale. The separation of scales breaks down when $T$ is a few hundred $\mathrm{MeV}$, since $g \sim 1$ in that temperature range, and these EFTs also become ineffective.

However, this is precisely the range of temperatures which is of great physical interest. The finite temperature crossover from a chiral symmetry broken hadronic state to a symmetry restored quark-gluon state occurs here. It is also the range of temperatures which seems to be most relevant for experiments using heavy-ion collisions. Some models have been used to explore the physics of this region and have had moderate success in matching lattice computations $[4,5]$.

Here we investigate a related model EFT which is built to describe the physics of QCD at finite temperature, around the crossover temperature $T_{\mathrm{co}}$. We follow a method which

\footnotetext{
sgupta@theory.tifr.res.in

†rishi@theory.tifr.res.in
}

Published by the American Physical Society under the terms of the Creative Commons Attribution 4.0 International license. Further distribution of this work must maintain attribution to the author(s) and the published article's title, journal citation, and DOI. Funded by SCOAP ${ }^{3}$. is often used when there is very limited information about the system under study [1]. Then one can write an EFT by guessing what the relevant fields and global symmetries are, and then writing down all possible terms in the Lagrangian which are constrained by the relevant global symmetries. Since we already know the full theory, namely QCD, it may seem that this process is less than optimal, since it does not exploit all the knowledge that the theory contains. The problem in deriving an effective theory from QCD, however, is that there is no known small parameter which can be exploited to do this accurately.

The model is written down in Sec. II. The mean field theory (MFT) is briefly examined in Sec. III. Fluctuations around the MFT are considered in Sec. IV. The description of lattice measurements is taken up in Sec. V. The regularization of integrals is discussed in the Appendix. These are technical parts of the paper. A nontechnical discussion is in the concluding Sec. VI; it is possible to read this section before the rest of the paper.

\section{THE EFFECTIVE THEORY MODEL}

The global symmetries of QCD which we use to build an EFT model are the vector (V) and axial (A) flavor symmetries $\mathrm{SU}_{V}\left(N_{f}\right) \times \mathrm{SU}_{A}\left(N_{f}\right)$, for $N_{f}$ flavors of quarks. We will build a model of interacting quark fields designed to match physics near some finite temperature $T_{0}$. The model is organized by mass dimension of operators, using the intrinsic scale $T_{0}$ to give dimensions to couplings where necessary. The fermion fields carry Dirac and flavor indices. We carry along the $\mathrm{SU}\left(N_{c}\right)$ color index, although these contribute only overall factors since there are no color interactions in the model: every fermion bilinear we build is color blind. We have no a priori argument for this, but proceed on this assumption to examine phenomena. We use the notation $\mathcal{N}=4 N_{c} N_{f}$ for the dimension of the fermion 
field, choosing $N_{c}=3$ and $N_{f}=2$ in this paper. The extension to $N_{f}=3$ is interesting and will be examined in the future.

Euclidean Dirac matrices are chosen to be Hermitian, with $\gamma_{4}=-i \gamma_{0}$, and $\gamma_{5}=\gamma_{1} \gamma_{2} \gamma_{3} \gamma_{4}$. The conjugate fermion field is defined as $\bar{\psi}=\psi^{\dagger} \gamma_{4}$. Since the Lorentz group becomes a rotation group, its generators are Hermitian, $S_{\mu \nu}=$ $-i\left[\gamma_{\mu}, \gamma_{\nu}\right] / 4$. Since we model thermal physics, time and space components are distinguished. As a result the finite temperature theory breaks the full $\mathrm{O}(4)$ rotational symmetry to a cylindrical symmetry $\mathrm{O}(3) \times \mathrm{Z}_{2}$, where $\mathrm{O}(3)$ is the spatial rotational symmetry and $\mathrm{Z}_{2}$ is the Euclidean time reversal symmetry $\mathrm{T}$ [6]. Every $\mathrm{O}(4)$ tensor also reduces. A finite temperature effective field theory then has many more couplings than a zero temperature theory. In order to describe the thermodynamics of the original theory, it suffices to write only CPT (Charge conjugation, Parity, Time reversal) invariant terms in the EFT. Vector flavor transformations act on spinors as $\psi \rightarrow \exp \left(i \tau^{a} \theta^{a} / 2\right) \psi$, and the axial as $\psi \rightarrow \exp \left(i \tau^{a} \theta^{a} \gamma_{5} / 2\right) \psi$.

We will write the Lagrangian of the EFT without a vacuum energy term, $d^{0} T_{0}^{4}$. There are no terms of dimension- 1 or -2 . The dimension-3 operator $\bar{\psi} \psi$ has the quark pole mass as its coefficient, which we write as $m_{0}=d^{3} T^{0}$. The dimension- 4 terms are obtained by using derivative operators: $\bar{\psi} \not \partial_{4} \psi$ and $\bar{\psi} \not \partial \psi$. Here $\not_{4}=\gamma_{4} \partial_{4}$ and $\not \partial=\gamma_{i} \partial_{i}$. These are invariant under $\mathrm{P}$ and $\mathrm{T}$, and also $\mathrm{V}$ and A. Inserting further Dirac structures in the two bilinears makes them lose discrete symmetries. The coefficient of the kinetic term, $\bar{\psi} \not \partial_{4} \psi$ fixes the normalization of the field operator, and hence is always set to unity. The coefficient of the other term, $d^{4}$, is special to finite temperature.

In a quadratic theory of fermions, the poles of the inverse propagator would be the solution of $p_{4}^{2}+\left(d^{4}\right)^{2}|\mathbf{p}|^{2}+m_{0}^{2}=0$. Pole mass, $i m_{0}$, is the term used for the pole of the temporal correlator averaged over space, i.e., for $|\mathbf{p}|=0$. The screening mass is the pole of the static propagator, i.e., for $p_{4}=0$. So $d^{4} \neq 1$ is just the statement that screening and pole masses are not equal. A general limit on $d^{4}$ comes from the requirement that after Wick rotation the group velocity of a wave packet should be less than unity. In a quadratic theory this implies that $0<d^{4}<1$. With an UV cutoff, one can have larger values of $d^{4}$ without running into problems with causality.

Three terms of mass dimension-5 can be obtained by using derivatives: $\bar{\psi} \not \partial_{4} \not \partial_{4} \psi, \bar{\psi} \not \partial \not \partial \psi$, and $\bar{\psi} \not \partial \not \partial \psi$. All three are invariant under $\mathrm{C}, \mathrm{P}, \mathrm{T}$ and $\mathrm{V}$, but not under $\mathrm{A}$. One can restore A by putting extra Dirac matrices in the bilinears, but this will destroy some of the discrete spacetime symmetries. As a result, there are no dimension-5 terms in the model.

Terms of mass dimension- 6 can either be built using fermionic current-current interactions or using derivatives in fermion bilinears. Four terms of the second kind are $\bar{\psi} \not \partial_{4}^{3} \psi, \bar{\psi} \not \partial_{4}^{2} \not \partial \psi, \bar{\psi} \not \partial_{4} \not \partial^{2} \psi$, and $\bar{\psi} \not \partial \psi$. All three are invariant under $\mathrm{P}$ and $\mathrm{T}, \mathrm{V}$ and $\mathrm{A}$. One can use the equation of motion at dimension- 4 to remove $\not_{4}$ and reduce all terms to that involving only spatial derivatives. This term should be added to the effective theory. However, it affects neither the MFT nor the fluctuations at the order we examine. So we do not include it here. Inserting further Dirac structures in the two bilinears makes them lose the invariance under P, C or T. The four-Fermi terms are restricted by chiral invariance. We may add terms of higher dimension if required.

The Euclidean EFT model we start with then consists of all possible terms up to mass dimension- 6 , invariant under the global and space-time symmetries of a finite temperature Euclidean theory,

$$
\begin{aligned}
L= & d^{3} T_{0} \bar{\psi} \psi+\bar{\psi} \not \partial_{4} \psi+d^{4} \bar{\psi} \not \partial_{i} \psi+L_{6}, \quad \text { where } \\
L_{6}= & +\frac{d^{61}}{T_{0}^{2}}\left[(\bar{\psi} \psi)^{2}+\left(\bar{\psi} i \gamma_{5} \tau^{a} \psi\right)^{2}\right]+\frac{d^{62}}{T_{0}^{2}}\left[\left(\bar{\psi} \tau^{a} \psi\right)^{2}+\left(\bar{\psi} i \gamma_{5} \psi\right)^{2}\right]+\frac{d^{63}}{T_{0}^{2}}\left(\bar{\psi} \gamma \gamma_{4} \psi\right)^{2}+\frac{d^{64}}{T_{0}^{2}}\left(\bar{\psi} i \gamma_{i} \psi\right)^{2}+\frac{d^{65}}{T_{0}^{2}}\left(\bar{\psi} \gamma_{5} \gamma_{4} \psi\right)^{2} \\
& +\frac{d^{66}}{T_{0}^{2}}\left(\bar{\psi} i \gamma_{5} \gamma_{i} \psi\right)^{2}+\frac{d^{67}}{T_{0}^{2}}\left[\left(\bar{\psi} \gamma_{4} \tau^{a} \psi\right)^{2}+\left(\bar{\psi} \gamma_{5} \gamma_{4} \tau^{a} \psi\right)^{2}\right]+\frac{d^{68}}{T_{0}^{2}}\left[\left(\bar{\psi} i \gamma_{i} \tau^{a} \psi\right)^{2}+\left(\bar{\psi} i \gamma_{5} \gamma_{i} \tau^{a} \psi\right)^{2}\right] \\
& +\frac{d^{69}}{T_{0}^{2}}\left[\left(\bar{\psi} i S_{i 4} \psi\right)^{2}+\left(\bar{\psi} S_{i j} \tau^{a} \psi\right)^{2}\right]+\frac{d^{60}}{T_{0}^{2}}\left[\left(\bar{\psi} i S_{i 4} \tau^{a} \psi\right)^{2}+\left(\bar{\psi} S_{i j} \psi\right)^{2}\right] .
\end{aligned}
$$

This differs from the Nambu-Jona-Lasinio (NJL) model [4] in a few ways. First, it is built to model QCD at finite temperature, hence Lorentz invariance is not used, and a temperature scale $T_{0}$ is used to organize the expansion. Second, it is an EFT, so all terms up to a certain order in mass dimension are kept, provided they are invariant under the symmetries of the model. The NJL model would have all four-Fermi couplings set to zero except $d^{61}$. Some of the other couplings have been considered before [7].

For later use we point out a fact which is well known [8]. Since the dimension- 6 terms are specifically written to preserve both the vector and axial parts of the chiral symmetry, it is only the mass term which breaks the symmetry. We have chosen the mass matrix to be diagonal in flavor, so only the axial part of the chiral symmetry is 
broken by it. As a result, we have the following relation for the divergence of the axial current:

$$
\partial_{\mu} j_{\mu}^{a}(x)=2 d^{3} T_{0} P^{a}, \quad \text { where } P^{a}=\frac{1}{2} \bar{\psi} \gamma_{5} \tau^{a} \psi .
$$

This is the partially conserved axial current (PCAC) relation.

\section{THE MEAN FIELD THEORY}

The fermionic mean field approximation is the operator identity $\bar{\psi}_{\alpha} \psi_{\beta}=\delta_{\alpha \beta}\langle\bar{\psi} \psi\rangle$, where $\alpha$ and $\beta$ are composite Dirac-flavor-color indices. Performing the Wick contractions in various ways in the generic 4-Fermi term then gives

$$
\begin{aligned}
(\bar{\psi} \Gamma \psi)^{2}= & 2\langle\bar{\psi} \psi\rangle[\operatorname{Tr} \Gamma \bar{\psi} \Gamma \psi-\bar{\psi} \Gamma \Gamma \psi] \\
& -\langle\bar{\psi} \psi\rangle^{2}\left[(\operatorname{Tr} \Gamma)^{2}-\operatorname{Tr}(\Gamma \Gamma)\right] .
\end{aligned}
$$

The product of Dirac-flavor matrices in the second term is the Fierz transformation. Since all the Dirac matrices as well as the rotation generators are traceless, only $\Gamma=1$ contributes to the first term. Also, since $\gamma_{\mu}^{2}=1$ for all Euclidean Dirac matrices, we find that the second is proportional to the identity for all currents. Using this we can reduce the interactions to an effective mass term

$$
\begin{aligned}
L_{6} & =-\mathcal{N}\left(\frac{T_{0}^{2}}{4 \lambda}\right) \Sigma^{2}+\Sigma \bar{\psi} \psi, \text { where } \Sigma=\frac{2 \lambda}{T_{0}^{2}}\langle\bar{\psi} \psi\rangle \text { and } \\
\lambda & =(\mathcal{N}+2) d^{61}-2 d^{62}-d^{63}+d^{64}+d^{65}-d^{66}+d^{69}-d^{60} .
\end{aligned}
$$

Note that exactly the same result would have been obtained in the mean field approximation to the NJL model [4]. The NJL mean field theory is widely explored [9], and we only need to adapt the results to take into account Lorentz symmetry breaking. The EFT is

$$
\begin{aligned}
L_{\mathrm{MFT}} & =-\mathcal{N}\left(\frac{T_{0}^{2}}{4 \lambda}\right) \Sigma^{2}+\bar{\psi} \not \partial_{4} \psi+d^{4} \bar{\psi} \not \partial_{i} \psi+m \bar{\psi} \psi, \quad \text { where } \\
m & =d^{3} T_{0}+\Sigma
\end{aligned}
$$

is an effective quark mass. $T_{0}$ can be chosen as we wish. There are only three couplings in this model. These have to be determined from data. $\Sigma$ must come out of a computation. We use dimensional regularization (DR) to deal with this to deal with this theory. Details are given in the Appendix, which also contains the notation used in the remainder of the paper.

\section{A. $T_{0}$ and couplings}

Using the methods of the Appendix, we find that the free energy density in the MFT, using dimensional regularization, is

$$
\begin{aligned}
-\Omega= & \frac{\mathcal{N} T_{0}^{2} \Sigma^{2}}{4 \lambda}+\frac{\mathcal{N} m^{4}}{64 \pi^{2}\left(d^{4}\right)^{3}}\left[\log \left(\frac{m^{2}}{\left(d^{4}\right)^{2} M^{2}}\right)-\frac{3}{2}\right] \\
& +\frac{\mathcal{N} T}{2 \pi^{2}\left(d^{4}\right)^{3}} \int_{0}^{\infty} d p p^{2} \log \left[1+\exp \left(-\frac{E}{T}\right)\right],
\end{aligned}
$$

where $M$ is a scale from dimensional regularization and $E^{2}=p^{2}+m^{2}$, where $p$ is rescaled in the last term to include $d^{4}$, so giving rise to the Jacobian factor $1 /\left(d^{4}\right)^{3}$. The value of $\Sigma$ must be that which minimizes $\Omega$ for fixed $T$. This, and the gap equation, $d \Omega / d \Sigma=0$, are very similar to the usual solutions of the NJL model. Putting the value of $\Sigma$ at the minimum back into Eq. (6), one obtains the free energy density as a function of temperature, $\Omega_{0}$. The pressure is $P=-\Omega_{0}$.

In the chiral limit and at low temperature, the minimum is at nonvanishing $\Sigma$, whereas at high temperature $\Sigma=0$ is the only solution. The temperature at which the trivial solution becomes the minimum is the critical temperature, $T_{c}$. By taking the second derivative of $\Omega$ with respect to $\Sigma$ and requesting that this vanish at $T_{c}$, one finds

$$
\frac{\left(d^{4}\right)^{3}}{\lambda}=\frac{1}{12} \frac{T_{c}^{2}}{T_{0}^{2}}
$$

Since we are interested in the region where the system crosses over from one state to another, we can choose $T_{0}$ to be equal to $T_{c}$. We will distinguish $T_{c}$ from the crossover temperatures $T_{\text {co }}$ obtained away from the chiral limit. In this case $\lambda=12\left(d^{4}\right)^{3}$. Note that this is just a convention for $T_{0}$, and not a prediction of $T_{c}$. By changing the convention we would only shift the value of $\lambda /\left(d^{4}\right)^{3}$, while keeping $T_{c}$ unchanged.

Note that there are two more couplings to be determined, namely $d^{3}$ and $d^{4}$. We will have to use two observables in order to fix these. Note that unlike the combination $\lambda /\left(d^{4}\right)^{3}$, these may depend on the renormalization scale $M$. On changing $M$ one needs to change couplings in order to keep the observables unchanged. This is a renormalization group running for the couplings, although its validity is limited because $M$ cannot be made arbitrarily large. It may seem that a freedom to choose the coupling $\lambda$ to fit a third observation has gone away. This is not so; the freedom has been transmuted into a choice of the as yet unspecified dimensionful quantity $T_{0}$. A third observation is required to fix this coupling. Once this is done, everything else is a prediction.

\section{B. Curvature of the critical line}

One may add a chemical potential term to the action in the form $-\mu \bar{\psi} \gamma_{4} \psi$. It turns out then that the critical point in the chiral limit at $\mu=0$ develops into a critical line. The same computation as above, now done at small $\mu$ gives an equation for the critical line, 


$$
T_{c}(\mu)^{2}+\frac{3}{\pi^{2}} \mu^{2}=T_{0}^{2}
$$

in the chosen convention $T_{0}=T_{c}(0)$ and quite independent of the couplings in the theory. The curvature of the critical line in the chiral limit is usually given in terms of the expansion

$$
T_{c}(\mu)=T_{c}(0)-\frac{1}{2} \kappa \frac{\mu^{2}}{T_{c}(0)}+\mathcal{O}\left(\mu^{3}\right)
$$

Comparing these two equations, we find the parameter free prediction

$$
\kappa=\frac{3}{\pi^{2}}
$$

Estimates of this quantity have been made on the lattice with quarks which are somewhat heavier than those found in nature. The results correspond to the range $\kappa \simeq$ 0.01-0.05 [10]. Although the mean field prediction in the chiral limit is larger, it is not so far away that it cannot be improved by various corrections.

These include corrections due to fluctuations around the mean field, or couplings between the condensate and number density. Interestingly though, there are other corrections which may be larger. The perturbation to the action due to a chemical potential breaks $C P$ symmetry by a dimension-3 term. This hard breaking in the UV could generate other $\mathrm{CP}$-violating terms in the effective action. Enumerating and controlling them all is a problem we will address in the future.

\section{PIONIC FLUCTUATIONS}

\section{A. The pion field}

In order to examine fluctuations around the mean field solution of the fermionic model, we use the HubbardStratanovich trick and introduce a matrix valued field $V$ with composite Dirac, flavor and color indices to linearize the action in Eq. (1). The equation of motion for $V$ gives

$$
V_{\alpha \beta}=\psi_{\alpha} \bar{\psi}_{\beta}
$$

Since the dimension- 6 terms of the action have been constructed to be invariant under axial flavor transformations, simultaneous transformations of $V, \psi$ and $\bar{\psi}$ leave even the linearized form invariant.

Fluctuations in the axial direction about the condensate are therefore captured by local "isospin waves" parametrized in the form

$$
\psi \rightarrow \mathrm{e}^{i \pi^{a} \tau^{a} \gamma_{5} /(2 f)} \psi \quad \text { and } \quad \bar{\psi} \rightarrow \bar{\psi} \mathrm{e}^{i \pi^{a} \tau^{a} \gamma_{5} /(2 f)},
$$

where $\pi^{a}$ are bosonic fields, and $f$ an emergent constant of dimension-1. These fields drop out of the dimension- 6 terms, and are seen only in the dimension-4 terms. Since the path integral is now quadratic in the fermion fields, we integrate them out to one-loop order to get the tree-level action of the fluctuations up to dimension-4:

$$
L_{f}=\frac{c^{2} T_{0}^{2}}{2} \pi^{2}+\frac{1}{2}\left(\partial_{4} \pi\right)^{2}+\frac{c^{4}}{2}(\nabla \pi)^{2}+\frac{c^{41}}{8} \pi^{4} .
$$

The only possible contraction of $\mathrm{SU}(2)$ flavor indices for the quartic term is $\left(\pi^{2}\right)^{2}$. A similar theory without the quartic terms has been previously considered in [11]; in their notation $c^{2} T_{0}^{2}=m^{2}$ and $c^{4}=u^{2}$. The theory seems to contain four constants, namely $f$, and the couplings $c^{2}, c^{4}$ and $c^{41}$. However, the underlying fermionic theory from which we derive this has only three couplings. In a later section we will give the computation of the couplings in Eq. (13) to one-loop order. As with any EFT, higher dimensional terms may be systematically added to $L_{f}$ if needed.

The contribution of the fluctuations to the free energy from the tree-level action of Eq. (13) is straightforward, since it reduces to a computation only in the quadratic theory. Since this closely parallels the MFT computation, we write down the result,

$$
\begin{aligned}
\Omega_{\pi}= & \frac{3\left(c^{2} T_{0}^{2}\right)^{2}}{64 \pi^{2}\left(c^{4}\right)^{3 / 2}}\left[\log \left(\frac{c^{2} T_{0}^{2}}{c^{4} M^{2}}\right)-\frac{3}{2}\right] \\
& +3 T \int \frac{d^{3} p}{(2 \pi)^{3}} \log \left(1-\mathrm{e}^{-E / T}\right),
\end{aligned}
$$

where $E^{2}=c^{4} p^{2}+c^{2} T_{0}^{2}$ and $M$ is the scale which arises from dimensional regularization of the vacuum energy term. The thermal integral is well known.

\section{B. Current algebra and parameters of the quadratic action}

We first make a few remarks about symmetry relations using Eq. (13). At small momentum we have

$$
\int d^{4} x \mathrm{e}^{i q \cdot x}\left\langle\pi^{a}(x) \pi^{b}(0)\right\rangle=\frac{\delta^{a b}}{c^{2} T_{0}^{2}+q_{4}^{2}+c^{4} \mathbf{q}^{2}} .
$$

In the static limit one obtains

$\lim _{q^{4} \rightarrow 0} \int d^{4} x \mathrm{e}^{i q \cdot x}\left\langle\pi^{a}(x) \pi^{b}(0)\right\rangle=\frac{\delta^{a b}}{c^{4}} \times \frac{1}{\mathbf{q}^{2}+\left(c^{2} T_{0}^{2} / c^{4}\right)}$.

This implies that the pion screening mass is $M_{\pi}=T_{0} \sqrt{c^{2} / c^{4}}$. Use of an effective theory simplifies the computation of screening masses, a fact that has been used before [12]. 
Using the definition of the axial vector rotation by an angle $\theta^{a}$ on quark fields, and the definition of the pion field, one sees that the transformation acts on the pion field as

$$
\pi^{a}(x) \rightarrow \pi^{a}(x)+f \theta^{a} .
$$

The axial Noether currents for $L_{f}$ of Eq. (13) are then

$$
j_{4}^{a}(x)=f \partial_{4} \pi^{a}+\cdots \quad j_{i}^{a}(x)=f c^{4} \partial_{i} \pi^{a}+\cdots,
$$

where the dots represent terms that arise when higher dimensional terms are kept in $L_{f}$. As a result, current correlators can be written as

$$
\begin{aligned}
\left\langle j_{4}^{a}(x) j_{4}^{b}(y)\right\rangle & =f^{2}\left\langle\partial_{4} \pi^{a}(x) \partial_{4} \pi^{b}(y)\right\rangle+\cdots \\
\left\langle j_{i}^{a}(x) j_{i}^{b}(y)\right\rangle & =\left(f c^{4}\right)^{2}\left\langle\partial_{i} \pi^{a}(x) \partial_{i} \pi^{b}(y)\right\rangle+\cdots .
\end{aligned}
$$

The momentum space correlator of the axial charge density can now be written as

$$
\begin{aligned}
\int d^{4} x \mathrm{e}^{i q \cdot x}\left\langle j_{4}^{a}(x) j_{4}^{b}(0)\right\rangle & =f^{2} \delta^{a b} q_{4}^{2}\left\langle\pi^{a}\left(q_{4}, \mathbf{q}\right) \pi^{b}\left(-q_{4},-\mathbf{q}\right)\right\rangle \\
& =\frac{f^{2} \delta^{a b} q_{4}^{2}}{c^{2} T_{0}^{2}+q_{4}^{2}+c^{4} \mathbf{q}^{2}} \stackrel{q_{4} \rightarrow 0}{\longrightarrow} 0 .
\end{aligned}
$$

Since the static limit gives a vanishing result, screening correlators of this component of the current cannot be used to constrain the parameters of the effective theory.

For the correlator of the spatial part of the current, we have

$$
\begin{aligned}
& \int d^{4} x \mathrm{e}^{i q \cdot x}\left\langle j_{i}^{a}(x) j_{i}^{b}(0)\right\rangle \\
& =\left(f c^{4}\right)^{2} \delta^{a b} \mathbf{q}^{2}\left\langle\pi^{a}\left(q_{4}, \mathbf{q}\right) \pi^{b}\left(-q_{4},-\mathbf{q}\right)\right\rangle \\
& =\frac{\left(f c^{4}\right)^{2} \delta^{a b} \mathbf{q}^{2}}{c^{2} T_{0}^{2}+q_{4}^{2}+c^{4} \mathbf{q}^{2}} \stackrel{q_{4} \rightarrow 0}{\longrightarrow} \frac{f^{2} c^{4} \delta^{a b} \mathbf{q}^{2}}{\mathbf{q}^{2}+M_{\pi}^{2}} .
\end{aligned}
$$

This differs from the pion correlator only in the coefficient. Since the measurement of a single current correlation function can give both the coefficient and the screening mass, we can constrain the model entirely by the measurement of this correlation function.

Given the relations in Eqs. (2) and (18), one easily finds

$$
\begin{aligned}
& \int d^{4} x \mathrm{e}^{i q \cdot x}\left\langle P^{a}(x) P^{b}(0)\right\rangle \\
& =\left(\frac{f}{2 m_{0}}\right)^{2} \delta^{a b} \frac{\left(q_{4}^{2}+c^{4} \mathbf{q}^{2}\right)^{2}}{c^{2} T_{0}^{2}+q_{4}^{2}+c^{4} \mathbf{q}^{2}} \stackrel{q_{4} \rightarrow 0}{\longrightarrow} \frac{f^{2} c^{4}}{4 m_{0}^{2}} \times \frac{\mathbf{q}^{4} \delta^{a b}}{\mathbf{q}^{2}+M_{\pi}^{2}} .
\end{aligned}
$$

So the measurement of the static correlator for $P^{a}$ can also be used to find both the screening mass and a combination of the couplings.

It is useful to convert this to a form which can be directly implemented on the lattice. As long as the lattice cutoff is sufficiently larger than $T_{0}$, one can ignore the lattice spacing and treat it as a theory in a finite box with periodic boundary conditions. The screening correlators measured on the lattice are usually projected on to zero momentum in all directions except one. Fourier transforming such a momentum space correlator from Eq. (22) in a box of size $L$ with periodic boundary conditions gives contributions from both the poles with equal weight. Performing the integral then gives the screening correlator for $P^{a}$ to be

$$
C_{P}(z)=\frac{f^{2} c^{4} M_{\pi}^{3}}{4 m_{0}^{2}} \mathrm{e}^{-M_{\pi} L / 2} \cosh \left[M_{\pi}\left(\frac{L}{2}-z\right)\right] .
$$

With the aid of a finite temperature version of the GellMann-Oakes-Renner relation, which we show later, this can be put into a form which is directly measurable on the lattice. The screening correlator for the axial current polarized in the $z$-direction takes on the simple form,

$$
C_{J_{3}}(z)=\frac{1}{2} f^{2} c^{4} M_{\pi} \mathrm{e}^{-M_{\pi} L / 2} \cosh \left[M_{\pi}\left(\frac{L}{2}-z\right)\right] .
$$

This is also measurable on the lattice.

\section{Two-point functions}

We introduce the transformations, Eq. (12), into the MFT action, and then expand out the exponential to the lowest order needed for each of the following computations. The couplings needed in Eq. (13) can be obtained by computing the two-point and four-point functions using the processes shown in Figs. 1 and 2, respectively. These Feynman diagrams can be evaluated using the quark propagator obtained from the MFT and pion-quark couplings from the expansion of the exponential as explained here.

The two-point function can be written in momentum space as

$$
\begin{aligned}
S_{\pi}= & \frac{\mathcal{N}}{8 f^{2}} \int \frac{d^{4} q}{(2 \pi)^{4}} \tilde{\pi}^{a}\left(q_{\mu}\right) \tilde{\pi}^{a}\left(-q_{\mu}\right)\left[-q_{4} q_{4} \mathcal{I}_{44}\left(q_{\mu}\right)\right. \\
& \left.-q_{i} q_{i} \mathcal{I}_{i i}\left(q_{\mu}\right)+\mathcal{I}\left(q_{\mu}\right)\right],
\end{aligned}
$$
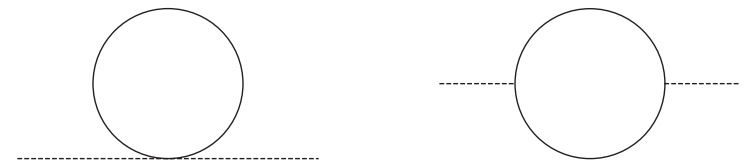

FIG. 1. Feynman diagram topologies for the two-point function of pions obtained by integrating out the quarks. Dashed lines stand for pions, full lines for quarks. 


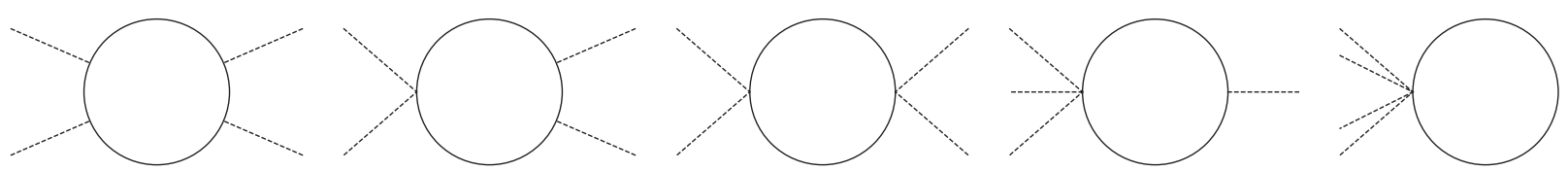

FIG. 2. Feynman diagram topologies for the four-point function of pions obtained by integrating out the quarks up to one-loop order. Dashed lines stand for pions, full lines for quarks.

where the integrals come from the one-loop Feynman diagrams shown in Fig. 1. The explicit forms of the integrals are

$$
\begin{aligned}
\mathcal{I}_{44}(q) & =\frac{1}{4} \int \frac{d^{4} p}{(2 \pi)^{4}} \operatorname{Tr}\left[\frac{1}{-i \not p+m}\left(\gamma^{5} \gamma^{4}\right) \frac{1}{-i \not \not^{\prime}+m}\left(\gamma^{5} \gamma^{4}\right)\right], \\
\mathcal{I}_{i i}(q) & =\frac{\left(d^{4}\right)^{2}}{4} \int \frac{d^{4} p}{(2 \pi)^{4}} \operatorname{Tr}\left[\frac{1}{-i \not p+m}\left(\gamma^{5} \gamma^{i}\right) \frac{1}{-i \not \not^{\prime}+m}\left(\gamma^{5} \gamma^{i}\right)\right], \\
\mathcal{I}(q) & =\frac{m_{0}^{2}}{4} \int \frac{d^{4} p}{(2 \pi)^{4}} \operatorname{Tr}\left[\frac{1}{-i \not p+m}\left(i \gamma^{5}\right) \frac{1}{-i \not p^{\prime}+m}\left(i \gamma^{5}\right)\right] \\
& +\frac{m_{0}}{4} \int \frac{d^{4} p}{(2 \pi)^{4}} \operatorname{Tr}\left[\frac{1}{-i \not p+m}\right]
\end{aligned}
$$

where $p^{\prime}=p+q$ and the trace is over spinor indices.

Taking the limit $q_{\mu} \rightarrow 0$ in the integrals allows us to match $S_{\pi}$ to the effective action in Eq. (13). This identification then implies the matching conditions:

$$
f^{2}=-\frac{\mathcal{N}}{4} I_{44}(0), \quad c^{4}=\frac{\mathcal{I}_{i i}(0)}{\mathcal{I}_{44}(0)}, \quad c^{2} T_{0}^{2}=-\frac{4 \mathcal{I}(0)}{\mathcal{I}_{44}(0)} .
$$

We write $p=\left(p^{4}, \mathbf{p}\right)$ and recall that $p^{4}=(2 n+1) \pi T$. Using the definition of the sum integral, as before, we have

$$
\begin{aligned}
\mathcal{I}_{44}(0)= & -T \sum_{n=-\infty}^{\infty} \int \frac{d^{3} p}{(2 \pi)^{3}} \frac{\left(p^{4}\right)^{2}-\left(d^{4}\right)^{2} \mathbf{p}^{2}+m^{2}}{\left(p^{2}+m^{2}\right)^{2}}, \\
\mathcal{I}_{i i}(0)= & -\left(d^{4}\right)^{2} T \sum_{n=-\infty}^{\infty} \int \frac{d^{3} p}{(2 \pi)^{3}} \\
& \times \frac{2\left(d^{4}\right)^{2} \mathbf{p}_{i} \mathbf{p}_{i}-\left(p^{4}\right)^{2}-\left(d^{4}\right)^{2} \mathbf{p}^{2}+m^{2}}{\left(p^{2}+m^{2}\right)^{2}} \\
\mathcal{I}(0)= & -m_{0}\left(m_{0}-m\right) T \sum_{n=-\infty}^{\infty} \int \frac{d^{3} p}{(2 \pi)^{3}} \frac{1}{p^{2}+m^{2}}
\end{aligned}
$$

After performing the sums in $p^{4}$ using the methods of [13] the integrands can be split into a thermal part and a part that is independent of $T$. The thermal parts of the integrals are exponentially damped for momenta much larger than $T$, and hence have no UV divergences. However, they cannot be exhibited in closed form, and are best evaluated numerically. We find that the integrals are

$$
\begin{aligned}
\mathcal{I}_{44} & =\frac{m^{2}}{4 \pi^{2}\left(d^{4}\right)^{3}} \log \left(\frac{m}{d^{4} M}\right)+\int \frac{d^{3} p}{(2 \pi)^{3}}\left[\frac{m^{2}}{E^{3}(\exp (E / T)+1)}-\frac{\left(d^{4}\right)^{2} \mathbf{p}^{2} \exp (E / T)}{E^{2} T(\exp (E / T)+1)^{2}}\right], \\
\mathcal{I}_{i i} & =\frac{m^{2}}{8 \pi^{2} d^{4}}\left[2 \log \left(\frac{m}{d^{4} M}\right)-\frac{1}{3}\right]-\int \frac{d^{3} p}{(2 \pi)^{3}}\left[\frac{\left(d^{4}\right)^{4} \mathbf{p}^{2}\left(1-\cos ^{2} \theta\right)}{E^{3}(\exp (E / T)+1)}-\frac{\left(\left(d^{4}\right)^{4} \mathbf{p}^{2} \cos ^{2} \theta+\left(d^{4}\right)^{2} m^{2}\right) \exp (E / T)}{E^{2} T(\exp (E / T)+1)^{2}}\right], \\
\mathcal{I} & =\frac{m_{0}\left(m_{0}-m\right) m^{2}}{16 \pi^{2}\left(d^{4}\right)^{3}}\left[1-2 \log \left(\frac{m}{d^{4} M}\right)\right]+\int \frac{d^{3} p}{(2 \pi)^{3}} \frac{m_{0}\left(m_{0}-m\right)}{E(\exp (E / 2 T)+1)}
\end{aligned}
$$

The vacuum terms are regulated in DR and $M$ is the $\overline{\mathrm{MS}}$ scale defined in the Appendix.

It can be shown that $c^{4}$ vanishes at $T_{c}$ in the chiral limit. Since the vacuum part of $\mathcal{I}_{i i}$ vanishes for $m \rightarrow 0$, we obtain

$$
\begin{aligned}
\mathcal{I}_{i i}(0)= & -\frac{1}{6 \pi^{2} d^{4}} \int_{0}^{\infty} \frac{d p p^{2}}{\exp (p / T)+1} \\
& \times\left[\frac{2}{p}-\frac{1}{T\{\exp (-p / T)+1\}}\right] .
\end{aligned}
$$

In terms of the variable $y=p / T$, the integral can be written as

$$
\begin{aligned}
\mathcal{I}_{i i}(0) & =-\frac{T^{2}}{6 \pi^{2} d^{4}}\left(I_{1}-I_{2}\right), \quad \text { where } \\
I_{1} & =2 \int_{0}^{\infty} \frac{d y y}{\mathrm{e}^{y}+1}, \\
I_{2} & =\int_{0}^{\infty} \frac{d y y^{2}}{\left(1+\mathrm{e}^{y}\right)\left(1+\mathrm{e}^{-y}\right)} .
\end{aligned}
$$

Interestingly, both $I_{1}$ and $I_{2}$ are equal to $\pi^{2} / 6$, as a result of which $\mathcal{I}_{i i}(0)$ vanishes. The cancellation can also be seen in the following way. $I_{1}$ has a set of single poles, and $I_{2}$ has double poles at exactly the same set of points. Furthermore, the residues at the poles exactly cancel between the two integrals. Since $\mathcal{I}_{i i}(0)=0$, by Eq. (27) $c_{4}$ vanishes at $T_{c}$ in 
the chiral limit [11]. Then, from the equation of motion, one sees that the pion field just becomes a constant nonpropagating field: the pion disappears above the critical point in the chiral limit. This creates no pathologies for the one-loop computation. However, in pion loops, thermal integrals are not UV regulated. This means that near $T_{c}$ higher dimensional terms must be added to Eq. (13) in order to make sense of the pion EFT.

Within the one-loop computation it is possible to ask how $c^{4}$ approaches zero near the chiral critical point. The argument above implies that the thermal part of $\mathcal{I}_{i i}$ varies as $m^{2}=\Sigma^{2}$. However, when $\Sigma$ is not exactly zero, the $T=0$ part must also be taken into account, and adds a $m^{2} \log m$ term. At the same time, $\mathcal{I}_{44}$ goes to a constant. From the gap equation we see that $\Sigma$ goes to zero at $T_{c}$ with a power behavior $\sqrt{1-T / T_{c}}$. However, a more careful examination shows that this is modified by a $\sqrt{1-T / T_{c}} \log \left(1-T / T_{c}\right)$ term. If one drops the logarithms, one would see $c^{4} \propto\left(1-T / T_{c}\right)$ [11]. The logarithms make this vanish slower, giving a leading behavior $c^{4} \propto\left(1-T / T_{c}\right) \log \left(1-T / T_{c}\right)$.

\section{Thermal Gell-Mann-Oakes-Renner relation}

A useful formal relation is called the Gell-Mann-OakesRenner (GMOR) relation. For small $m_{0}$ in Eq. (28), we can neglect the term quadratic in $m_{0}$, and write

$$
\mathcal{I}(0)=2 m_{0} \int \frac{d^{4} p}{(2 \pi)^{4}} \frac{m}{p^{2}+m^{2}},
$$

where the overall factor of 2 on the right comes from the fact that the 4-d integral is defined by a sum over positive Matsubara modes, but $\mathcal{I}$ is defined by a sum over all Matsubara modes. Next, using Eq. (27) we obtain

$$
c^{2} T_{0}^{2}=\frac{\mathcal{N}}{f^{2}} \mathcal{I}(0)=\frac{2 m_{0} \mathcal{N}}{f^{2}} \int \frac{d^{4} p}{(2 \pi)^{4}} \frac{m}{p^{2}+m^{2}} .
$$

The gap equation in the MFT shows that the integral is exactly the negative of $\langle\bar{\psi} \psi\rangle / 2$. As a result, we obtain the extension of the GMOR relation to finite temperature,

$$
c^{2} T_{0}^{2}=-\frac{\mathcal{N} m_{0}\langle\bar{\psi} \psi\rangle}{f^{2}} .
$$

The factor of $\mathcal{N}$ is conventional; it is often absorbed into the definition of the condensate. Since all the quantities appearing here have been defined in terms of the couplings in the fermion theory, this identity is a statement of selfconsistency at one-loop order. Note that when $m_{0}=0$ the identity implies $c^{2}=0$.

\section{E. Four-point functions}

Symmetry arguments prevent dimension-3 operators from appearing in the pion effective action of Eq. (13). Examination of the Feynman diagrams which appear to one-loop order in the computation of three-point functions gives the same result. Technically this happens because we need to take the trace of Dirac structures such as $\gamma_{5}, \gamma_{5} \gamma_{\mu} \gamma_{\nu}$ or $\gamma_{5} \gamma_{\mu} \gamma_{\nu} \gamma_{\rho}$, all of which vanish.

The coupling $c^{41}$ in Eq. (13) is computed from the Feynman diagrams shown in Fig. 2. Since every vertex in these diagrams carries a factor of $m_{0}$, the leading term in the chiral limit comes from the last diagram. This is easily evaluated, and gives

$c^{41}=\frac{\mathcal{N} m_{0}}{3 f^{4}} \int \frac{d^{4} p}{(2 \pi)^{4}} \frac{m}{p^{2}+m^{2}}=\frac{\mathcal{N} m_{0}\langle\bar{\psi} \psi\rangle}{3 f^{4}}=-\frac{m_{\pi}^{2}}{3 f^{2}}$.

We have used the GMOR relation to get the final form. The evaluation of the other diagrams is straightforward but tedious.

\section{DESCRIBING LATTICE COMPUTATIONS}

\section{A. Lattice data set}

We make a few remarks about the lattice computations which we use. A recent work [14] reported two sets of computations of correlators of the axial current as well as of the pseudoscalar isoscalar density. One set (called $\mathrm{C} 1$ in [14]) uses a quark mass which gives the crossover temperature $T_{\text {co }}=211 \pm 5 \mathrm{MeV}$. The other set (D1) has a lighter quark and gives $T_{\text {co }}=193 \pm 7 \mathrm{MeV}$. In each scan the temperature is measured with an accuracy of $2 \mathrm{MeV}$. However, in the set D1 the temperature scan below $T_{\text {co }}$ mostly covers a range of about $10 \mathrm{MeV}$ of the central value for $T_{\mathrm{co}}$, and hence is statistically indistinguishable from $T_{\text {co. }}$. As a result, we are forced to use the set called C1, which covers a larger temperature range.

Since the EFT is treated in dimensional regularization, loops give only logarithms of $m / M(M$ is the regularizing scale). However, a lattice regularization not only has the corresponding logs of $m a$ (where $a$ is the lattice spacing), but also powers. In the past decade it was realized that much faster convergence to continuum results are obtained by subtracting power corrections [15]. However, subtraction of power corrections has not yet been performed for two-point functions. Once lattice computations start doing this, a matching with the EFT will yield continuum results when $T \ll 1 / a$.

Comparing our expression for the axial current correlator in Eq. (24) with that used in [14], we find that what we call $f \sqrt{c^{4}}$ is called $f_{\pi}$ there. Also, what we call the screening mass, $M_{\pi}=T_{0} \sqrt{c^{2} / c^{4}}$, is called $m_{\pi}$ there, and the definition of the chiral condensate there corresponds to $-\mathcal{N}\langle\bar{\psi} \psi\rangle$ in our notation. With these translations, the 
results reported in [14] can be used with the expressions we use. For example, the GMOR relation is the same, since the factors of $\sqrt{c^{4}}$ cancel. Also, the definition of the quantity called $u_{f}$ in [14] is what we call $u$.

\section{B. Fits}

Since we do not have access to the covariances of the lattice computations our treatment of the errors is forcibly simplistic. We take the errors in $M_{\pi}$ and $c^{4}$ to be independent, and make four sets of fits at the extreme values allowed for each of these. Similarly, we take the error in $T_{\text {co }}$ to be completely independent of this. Also, since we require the dimensionless ratio $T / T_{\mathrm{co}}$, and we have no access to possible covariances between the numerator and denominator, we add the errors in quadrature. If lattice collaborations make these fits in the future, then all the covariances of the inputs can be taken into account.

We remarked earlier that there are three parameters of the EFT to be fitted. Two of these are the dimensionless couplings $d^{3}$ and $d^{4}$. The third coupling is dimensionally transmuted to the value of the critical temperature $T_{c}$, which has been chosen to coincide with $T_{0}$. The inputs which we use to fix these are the values of $M_{\pi}$ and $c^{4}$ (called $m_{\pi}$ and $u_{f}$ in [14]) at $T / T_{\text {co }}=0.84 \pm 0.02$ (corresponding to $T=177 \pm 2 \mathrm{MeV}$ ) and the value of the crossover temperature $T_{\text {co }} / T_{0}$, We define $T_{\text {co }}$ in the EFT to be the temperature at which the chiral susceptibility peaks.

The results of our fits are shown in Fig. 3. Little can be said directly from the values of the couplings, except that they seem to be of order unity. Since $d^{3} \rightarrow 0$ is the chiral limit, this indicates that the quark mass of the input data set is rather high. We have checked that the values of $d^{4}$ obtained in the fit do not violate causality.

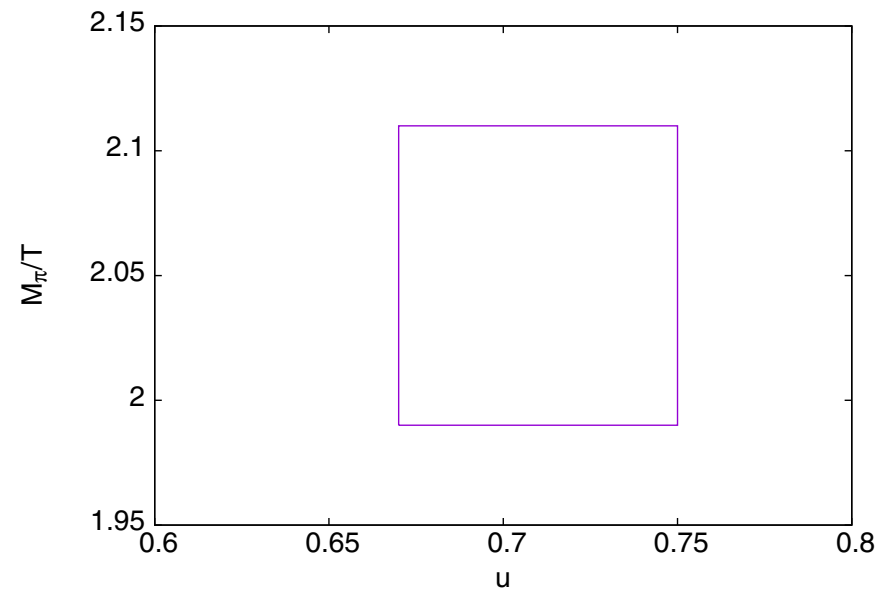

The best fit gives $T_{\text {co }} / T_{0}=1.24 \pm 0.03$. Since the lattice computation has $T_{\text {co }}=211 \pm 5 \mathrm{MeV}$, this yields $T_{0}=170 \pm 6 \mathrm{MeV}$. This is also the critical temperature in the chiral limit. The value agrees with the chiral extrapolations using the same action reported in [16].

\section{Checks and predictions}

The EFT becomes useful if the couplings $d^{3}, d^{4}$ and $\lambda$ vary little with temperature. Then the fit shown in Fig. 3 can be used to extract physics at a range of temperatures near $T_{\text {co }}$. The first check of whether this can be done is to examine the temperature dependence of $M_{\pi}$ and $c^{4}$, while keeping the fermionic couplings independent of $T$. This test is shown in Fig. 4. While the agreement is not perfect, the trend seen in the lattice computation seems to be reasonably well reproduced in the EFT. The dependence of the EFT predictions on the scale $M$ is seen to be small. There is some jitter in the lattice computations which could perhaps be removed if one uses larger statistics. Also, since the lattice spacing changes as $T$ is changed, at such large quark masses power corrections could modify the temperature dependence somewhat.

Independent tests of the model are the prediction of the other low-energy constants: $f$ and $c^{41}$. Since the model parameters are now fixed, there is no further freedom in these predictions. A comparison of our prediction with the lattice results for $f$ is shown in Fig. 5. The agreement is pleasant. There are no lattice computations of the four-point function of pions, so the prediction shown in Fig. 5 cannot be tested now. The dependence of these quantities on the scale $M$ is still mild, although a little larger than for $M_{\pi}$ and $c^{4}$.

From these checks it seems that the approximation of neglecting the temperature dependence of the couplings in the fermion EFT works fairly well in a range of temperature

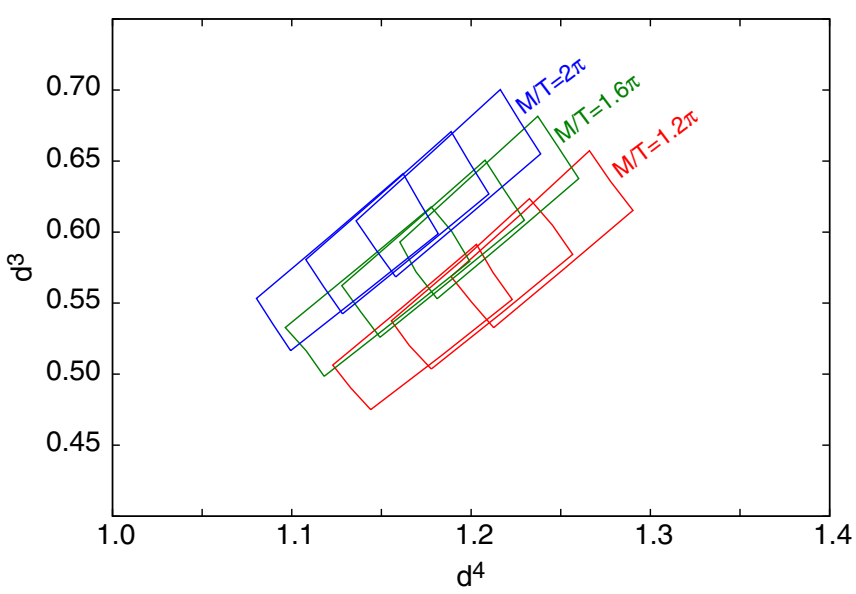

FIG. 3. The 1- $\sigma$ limits of the lattice results of [14] at $T / T_{\text {co }}=0.84 \pm 0.02$ define the input rectangular area in the panel on the left. The fitted values of the EFT couplings are given in the figure on the right. Traversing the rectangle on the left in the clockwise direction, one traverses the output rectangles also in the same sense. The fits depend on the values of the regularization scale $M$ as shown. For each $M$ we have shown three rectangles, these are for the central and upper and lower $1-\sigma$ values of $T_{\text {co }}$. 

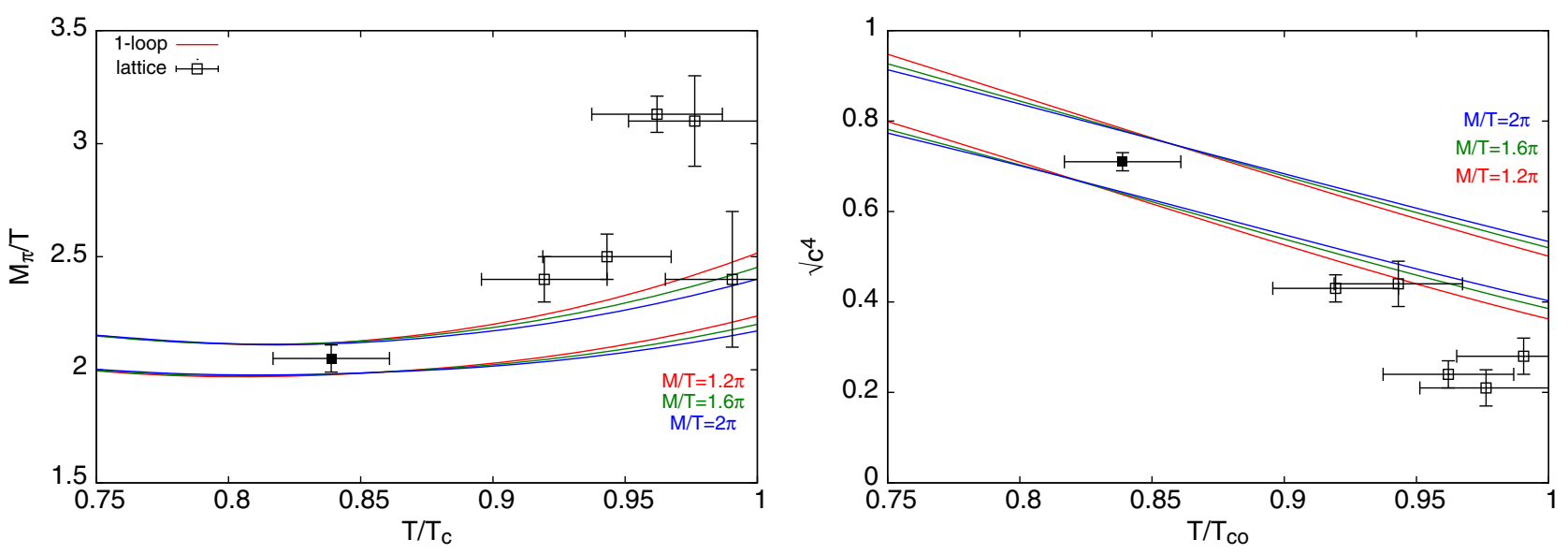

FIG. 4. Fits to lattice measurements of static pion correlators at finite temperature. The lattice measurements which are inputs to the fits are shown with filled symbols. Everything else is a check of the EFT. The predicted uncertainty bands are shown for different values of $M / T$. The legend for $M / T$ on the right edge of the figures shows the ordering of the bands, from top to bottom.

around $T_{c}$. The renormalization scale dependence of physical results is generally smaller than the uncertainty due to the errors in the input quantities.

However, the results on $c^{41}$ shown in Fig. 5 put limits on the use of Eq. (13) to compute pion loop corrections in future. Negative $c^{41}$ means that we are able to treat only fluctuations of magnitude $\pi<T_{0} \sqrt{c^{2} /\left|c^{41}\right|}$. In order to do pion loop integrals one must take into account higher dimension terms which stabilize the path integral over pions. We also see that at $T \simeq 0.75 T_{c}$ the coupling $c^{4}$ begins to approach unity. So it is possible at $T \leq 0.75 T_{\mathrm{co}}$, an effective theory tuned at $T=0$ may become a quantitatively useful tool.

Subject to this limitation, we can now compute extensive thermal quantities in the effective theory of fluctuations given in Eq. (13). This theory is constructed to be valid for $p<T$. For thermal quantities whose integrands are dominated by momenta in this region, one should get accurate predictions. Since thermal integrals have UV cutoffs $\exp \left(-E_{p} / T\right)$, we require $T<\sqrt{c^{4} T^{2}+c^{2} T_{0}^{2}} \simeq T \sqrt{c^{4}+c^{2}}$, where we have taken $T_{0} \simeq T$. However, for very small quark masses, near the crossover, where both $c^{4}$ and $c^{2}$ are small, the one-loop computation of fluctuations cannot give thermodynamic quantities accurately. This is connected with the fact that the pion becomes nonpropagating at the critical point in the chiral limit.

In the case we are examining, the convergence criterion is satisfied. Even so, some quantities are predicted more accurately than others. An examination of the integrals shows that the energy density, $E / T^{4}$, or the entropy density $S / T^{3}$, are less well controlled than the pressure, $P / T^{4}$. On the other hand the chiral susceptibility is better controlled. Recall also that the pressure is dominated by the lightest particle, and this must appear in the EFT. Therefore the pressure can be fairly well described as long as the technical criteria discussed here are satisfied. However
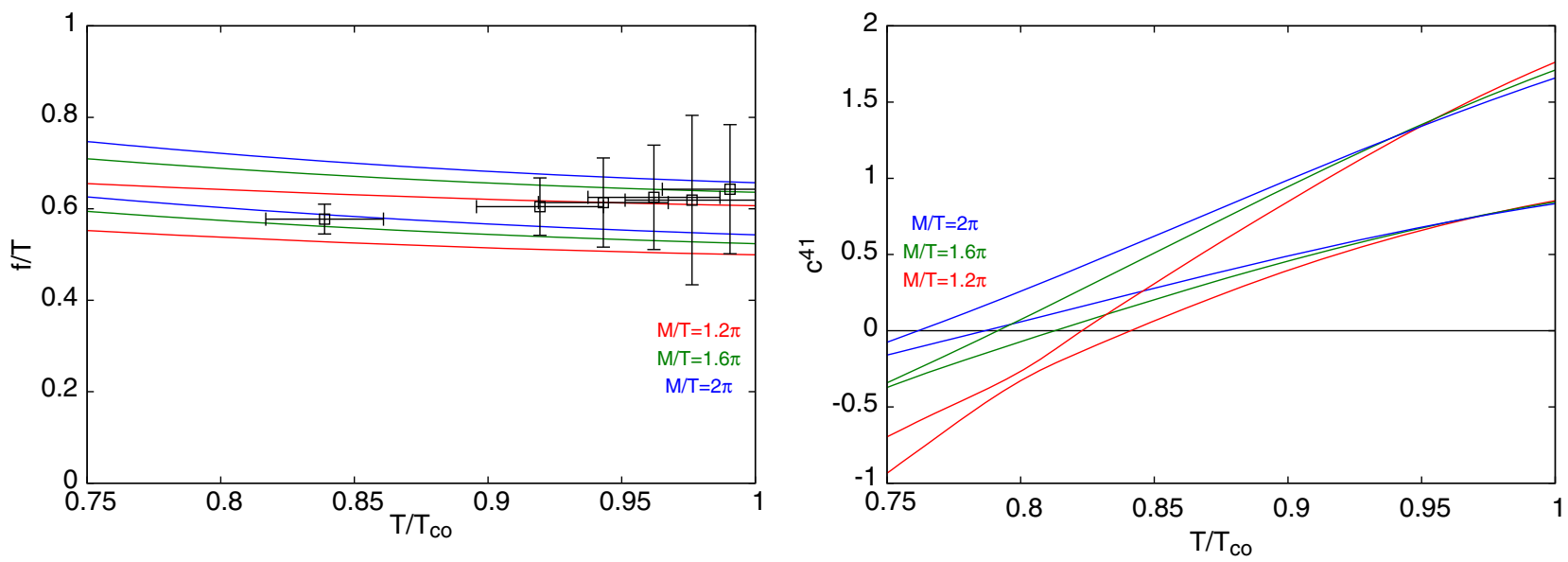

FIG. 5. From the fitted values of the fermion couplings, other pionic couplings can be predicted. The model predictions of $f / T$ and $c^{41}$ are shown in the two panels. Lattice computations of $f / T$ are compared to the EFT predictions. 


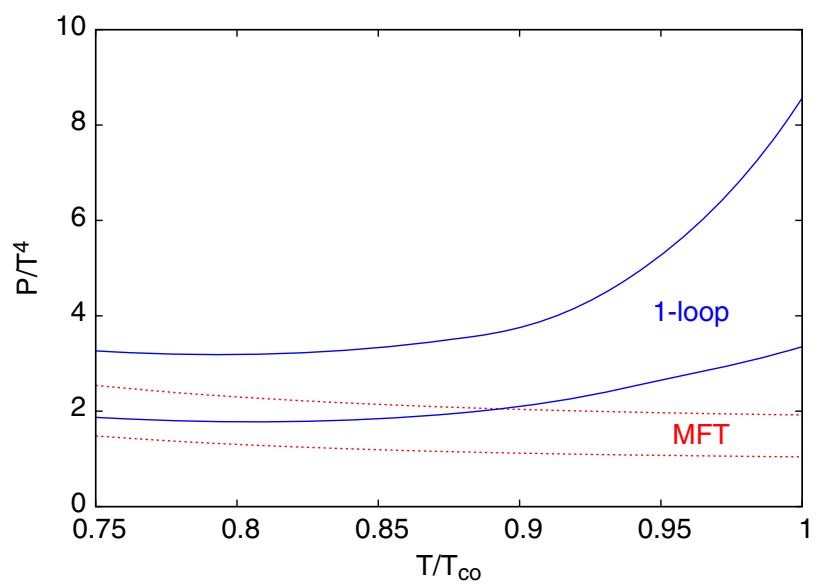

FIG. 6. From the fitted values of the fermion couplings, thermodynamic quantities can be predicted. The model prediction of the pressure, $P / T^{4}$, is shown here.

the energy density can get large contributions from massive modes which are not part of the EFT.

In Fig. 6 we show the pressure computed in the MFT and to one-loop order using Eq. (14). The rise seen in the oneloop correction comes from a factor of $\left(c^{4}\right)^{-3 / 2}$ obtained by doing the momentum integrals in the thermal pion contribution. Since this is a generic feature of the EFT model, so must be the increase in pressure as one approaches $T_{\mathrm{co}}$.

\section{DISCUSSION}

In this paper we developed an effective theory for strongly interacting matter with the modest aim of describing long-distance physics in a small range of temperatures around the QCD crossover temperature $T_{\mathrm{co}}$. This introduces a scale $T_{0} \simeq T_{\text {co }}$ for the temperature. Long-distance physics means momenta less than the temperature.

Since QCD at finite quark mass has a crossover, there is no sharp change in the nature of the degrees of freedom. On one side of the crossover, it could be natural and easy to use hadronic degrees of freedom, just as it is natural and easy to use quark and gluon degrees of freedom on the other side. However, the free energy has no singularity, and one should be able to push either description across the crossover, perhaps with some increase in the complexity of description.

In this spirit, in Sec. II we wrote down the most general Euclidean effective theory of quarks at finite temperature including dimension- 6 terms which are constrained by the chiral $\mathrm{SU}(2) \times \mathrm{SU}(2)$ symmetry of QCD. Although the action in Eq. (1) is a generalization of the NJL model, it contains some new features such as the difference between screening and pole masses. It also includes some extra couplings which have been considered in the literature from time to time. An obvious criticism is that we leave out gluons. We are unable to give a field theoretical justification for this. Since the model works well enough, as we showed quantitatively, one should turn this question around and ask what this implies for possible derivations of effective models from QCD.

The mean field theory is examined in Sec. III. The ten couplings for 4-Fermi operators reduce to one in this limit, leaving three couplings to be determined. Divergent integrals are treated in dimensional regularization (see the Appendix). This has a feature which is useful for thermal physics, namely that it regulates only the integrals which are divergent, but not the convergent finite temperature pieces. The gap equation completely fixes one combination of the couplings, as shown in Eq. (7), and by dimensional transmutation leaves the transition temperature in the chiral limit, $T_{c}$, as the third quantity to be fixed by data.

There is a critical line in the phase diagram at finite chemical potential in the chiral limit, whose curvature is predicted in MFT; see Eq. (10). This is within an order of magnitude of the same quantity computed on the lattice. We discussed in Sec. III that a chemical potential for quarks in the UV theory, namely $\mathrm{QCD}$, gives a hard breaking of $C P$ symmetry. This means that all operators which break this symmetry could enter into the EFT. An improved description of the phase diagram is therefore sufficiently complicated that we leave it to the future.

In Sec. IV, we examined low-energy isospin-wave fluctuations around the MFT. These are organized as another EFT written in terms of pion fields. All terms up to dimension-4 are given in Eq. (13). Through the Noether's theorem and PCAC we connected the couplings of the fermion EFT to lattice computations of two-point functions of currents in QCD. We demonstrated the oneloop computation of the couplings of this theory. We also showed that the Gell-Mann-Oakes-Renner (GMOR) relation remains valid at one-loop order in the EFT.

This model is applied to a description of lattice computations in Sec. V. All the parameters of the model are obtained by matching the EFT to lattice computations in [14] at one value of the temperature; the results are shown in Fig. 3. The lattice regularization contains power corrections in the quark mass, which should be subtracted in the future. Although the data available today do not perform these subtractions, the fits yield reasonable descriptions of the temperature dependence of the long-distance part of two-point functions of pions at all temperatures given in [14]. They also give predictions for as yet unmeasured fourpoint functions of pions; see Figs. 4 and 5. The pressure shows an interesting rapid rise below and close to $T_{\text {co }}$, which we have argued is a robust prediction of the model.

The EFT parameters give indications of limits on the model. One limit comes from the fact that the four-pion coupling $c^{41}$ becomes negative at about $0.75 T_{\text {co }}$, indicating that higher dimension terms are needed to stabilize pion loop corrections. At the same time, $c^{4}$ becomes of order unity, indicating that an EFT fitted at $T=0$ may be an appropriate computational tool at lower temperatures. Near 
and above $T_{\mathrm{co}}$, the falling value of $c^{4}$ indicates that higher derivative terms in the EFT may become necessary. This still leaves a window of applicability of the one-loop corrections to the MFT in terms of a pion EFT. The results we have shown are in this window.

Some future directions are clear. The pion EFT we derived at one-loop is very similar to that used in [11] for examination of departure from equilibrium. Similar realtime phenomena may be investigated in the fermion EFT which we work with. The question of the phase diagram is another direction, which we have already discussed. A parametrization of "radial" fluctuations can give us a better handle on the physics above $T_{\text {co }}$. The case $N_{f}=3$ will be interesting. So also will be a complete analysis of the model at finite chemical potential. One question that we have not examined at all is of the nonlinear sigma model which describes fluctuations. This can be interesting.

\section{APPENDIX: REGULARIZATION}

The free energy density for the MFT action in Eq. (5) can be written as $\Omega / \mathcal{N}=-T_{0}^{2} \Sigma^{2} /(4 \lambda)-I_{0}(m, T)$, where the integral

$$
\begin{aligned}
I_{0}(m, T) & =\int \frac{d^{4} p}{(2 \pi)^{4}} \log \left(p_{4}^{2}+p^{2}+m^{2}\right) \\
& =T \sum_{n} \int \frac{d^{3} p}{(2 \pi)^{3}} \log \left(\pi^{2}(2 n+1)^{2}+\frac{E_{p}^{2}}{T^{2}}\right),
\end{aligned}
$$

$E_{p}^{2}=\left(d^{4}\right)^{2} p^{2}+m^{2}$, and the sum over Matsubara modes goes only over positive $n$. This has a cubic divergence which needs to be cured. The origin of this divergence will be important to understand, since we expect no divergences in the thermodynamics of free fermions.

Using a standard trick [13] we can write

$$
\begin{aligned}
I_{0}(m, T) \\
=T \int \frac{d^{3} p}{(2 \pi)^{3}} \sum_{n}\left[\log \left(1+\pi^{2}(2 n+1)^{2}\right)\right. \\
\left.\quad+\int_{1}^{E_{p}^{2} / T^{2}} \frac{d y}{\pi^{2}(2 n+1)^{2}+y}\right] \\
=T \int \frac{d^{3} p}{(2 \pi)^{3}}\left[\sigma_{l}+\frac{1}{\pi^{2}} \int_{1}^{E_{p}^{2} / T^{2}} d y \sum_{n} \frac{1}{(2 n+1)^{2}+y / \pi^{2}}\right],
\end{aligned}
$$

where we have introduced the notation $\sigma_{l}$ for the temperature independent but divergent sum over the logarithmic terms. We may justify the interchange of the sum and the integral by putting an arbitrary UV cutoff at all stages until the cubic divergence in terms of this cutoff is removed and a finite result is obtained. After the change of variables, $t=\sqrt{y}$, and performing the remaining sum, one gets [13]

$$
I_{0}(m, T)=T \int \frac{d^{3} p}{(2 \pi)^{3}}\left[\sigma_{l}^{\prime}+\frac{E_{p}}{2 T}+\log \left(1+\mathrm{e}^{-E_{p} / T}\right)\right],
$$

where $\sigma_{l}^{\prime}$ absorbs all the factors independent of $p$ and $T$. Note that there are now two kinds of cubic divergences: one is linear in $T$ and comes from the integral over $\sigma_{l}^{\prime}$, the other is independent of $T$ and comes from the integral over $E_{p}$. The second term is just the zero-point energy of the fields, called the vacuum energy. The first term, which we may call the vacuum entropy, gives no contribution to derivatives of $\log Z=-F / T$, and hence cannot give any contribution to thermodynamics, and so may be subtracted out.

A cutoff regularization is easy to implement when it works, and cures all UV divergences. However, while it makes the vacuum entropy finite, it does not reduce it to zero. In view of this, a different regularization may be more useful. An alternative is to use a scheme which resembles Pauli-Villars regularization. In the familiar version of this regularization process one subtracts from a divergent integral another integral of the same form with $m \rightarrow M$ and choosing $M \gg m$. However, in this case that would still leave a linear divergence. We are forced to subtract more integrals to obtain a regular integral. This removes the vacuum entropy term. However the vacuum energy has powers of $m / M$.

In zero-temperature effective theories, dimensional regularization turns out to be very useful. At finite temperature one would have to work near $D=3$ spatial dimensions, since we want to regulate after doing the sum over all Matsubara frequencies. Recall that dimensional regularization needs a regularization scale $M$ which is used to give the correct dimension to the integral defined originally with $\epsilon=0$. The basic formula we need to use is

$$
\begin{aligned}
J_{n}^{m} & =\mu^{3-D} \int \frac{d^{D} k}{(2 \pi)^{D}} \frac{k^{2 m}}{\left(k^{2}+\ell^{2}\right)^{n}} \\
& =\ell^{2 m+D-2 n} \mu^{3-D} \frac{\Omega_{D}}{(2 \pi)^{D}} \frac{\Gamma(m+D / 2) \Gamma(n-m-D / 2)}{2 \Gamma(n)},
\end{aligned}
$$

where $\Omega_{D}=2(2 \pi)^{D / 2} / \Gamma(D / 2)$ is the volume of an unit sphere in $D$ dimensions. We set $D=3-2 \epsilon$.

For the vacuum entropy term, we have $m=0$ and $n=0$. The formula above shows that the integral is finite, and therefore zero due to its invariance under the choice of $\ell$. The vacuum energy term requires $m=0$ and $n=-1 / 2$. The formula then gives the following result: 
$J_{-1 / 2}^{0}=\left(\frac{\ell^{4}}{32 \pi^{2}}\right)\left[-\frac{1}{\epsilon}+\gamma-\frac{3}{2}+\log \left(\frac{\ell^{2}}{4 \pi \mu^{2}}\right)+\mathcal{O}(\epsilon)\right]$,

where $\gamma$ is the Euler-Mascheroni constant.

In order to compute the vacuum energy we scale $p \rightarrow\left(d^{4}\right) p$ in Eq. (A3). However, the regularization scale $\mu$ is a scale on $p$, so it must be scaled in the same way. Using the definition $M^{2}=4 \pi \mu^{2} \exp (-\gamma)$, and dropping the pole in $\epsilon$, we then get the MS result,

$$
I_{0}^{r}(m)=\left(\frac{m^{4}}{64 \pi^{2}\left(d^{4}\right)^{3}}\right)\left[\log \left(\frac{m^{2}}{\left(d^{4}\right)^{2} M^{2}}\right)-\frac{3}{2}\right] .
$$

Putting everything together, we find

$$
I_{0}(m, T)=I_{0}^{r}(m)+\frac{T}{\left(d^{4}\right)^{3}} \int \frac{d^{3} p}{(2 \pi)^{3}}\left[\log \left(1+\mathrm{e}^{-\sqrt{p^{2}+m^{2}} / T}\right)\right] .
$$

One can continue the thermal integral to arbitrary dimension. Since the integral is finite, taking the result to the limit $D \rightarrow 3$ gives no poles in $3-D$, and hence does not introduce the scale $M$ into the thermal integral. DR is therefore much more intuitive for the thermal part than either a cutoff or Pauli-Villars regularization would have been. One pleasant result of this is that large values of $\log (M / m)$ can be easily avoided, in the same way as at zero temperature. DR has been used before at finite temperature in [17].

Using Eq. (6), the gap equation, $d \Omega / d \Sigma=0$, becomes

$$
\begin{aligned}
\Sigma= & -\frac{2 \lambda}{T_{0}^{2}}\left\{\frac{m^{3}}{16\left(d^{4}\right)^{3} \pi^{2}}\left[\log \left(\frac{m^{2}}{\left(d^{4}\right)^{2} M^{2}}\right)-1\right]\right. \\
& \left.-\frac{m}{2\left(d^{4}\right)^{3} \pi^{2}} \int_{0}^{\infty} \frac{d p p^{2}}{E} \frac{1}{\exp (E / T)+1}\right\} .
\end{aligned}
$$

In the chiral limit, $d^{3}=0$ and so $m=\Sigma$. We can see then that $\Sigma=0$ is always a solution, and that there are generally two more real solutions related by sign flips.

Pion loop integrals may also be regularized in DR. We see that the convergent thermal parts of the integrals will give results of the form $T^{n} f\left(c^{2} T_{0} / T\right)$, where $n$ is the engineering dimension of the loop integral. At finite temperature this introduces a new scale in amplitudes, which would then modify the power counting of pion loops and give results different from those in, for example, [1]. This will be interesting at higher loop orders than what we examine.
[1] S. Weinberg, Phenomenological Lagrangians, Physica A (Amsterdam) 96A, 327 (1979).

[2] J. Gasser and H. Leutwyler, Thermodynamics of chiral symmetry, Phys. Lett. B 188, 477 (1987).

[3] K. Kajantie, M. Laine, K. Rummukainen, and Y. Schroder, Resumming Long-Distance Contributions to the QCD Pressure, Phys. Rev. Lett. 86, 10 (2001).

[4] Y. Nambu and G. Jona-Lasinio, Dynamical model of elementary particles based on an analogy with superconductivity. 1., Phys. Rev. 122, 345 (1961); Dynamical model of elementary particles based on an analogy with superconductivity. Ii, Phys. Rev. 124, 246 (1961).

[5] K. Fukushima, Chiral effective model with the Polyakov loop, Phys. Lett. B 591, 277 (2004); A. Dumitru and R. D. Pisarski, Degrees of freedom and the deconfining phase transition, Phys. Lett. B 525, 95 (2002).

[6] B. Grossman, S. Gupta, U. M. Heller, and F. Karsch, Glueball-like screening masses in pure SU(3) at finite temperatures, Nucl. Phys. B417, 289 (1994).

[7] T. Schäfer, E. V. Shuryak, and J. J. M. Verbaarschot, The chiral phase transition and instanton-anti-instanton molecules, Phys. Rev. D 51, 1267 (1995); V. Bernard, A. H. Blin, B. Hiller, Y. P. Ivanov, A. A. Osipov, and U. G. Meissner, Pion observables in the extended NJL model with vector and axial-vector mesons, Ann. Phys. (N.Y.) 249, 499 (1996);
M. K. Volkov and A. E. Radzhabov, The Nambu-JonaLasinio model and its development, Phys. Usp. 49, 551 (2006); Y. Sakai, K. Kashiwa, H. Kouno, M. Matsuzaki, and M. Yahiro, Vector-type four-quark interaction and its impact on QCD phase structure, Phys. Rev. D 78, 076007 (2008); A. V. Friesen, Y. L. Kalinovsky, and V. D. Toneev, Vector interaction effect on thermodynamics and phase structure of QCD matter, Int. J. Mod. Phys. A 30, 1550089 (2015).

[8] S. Weinberg, The Quantum Theory of Fields: Volume 2, Modern Applications (Cambridge University Press, Cambridge, 1996).

[9] S. P. Klevansky, The Nambu-Jona-Lasinio model of quantum chromodynamics, Rev. Mod. Phys. 64, 649 (1992).

[10] O. Kaczmarek, F. Karsch, E. Laermann, C. Miao, S. Mukherjee, P. Petreczky, C. Schmidt, W. Soeldner, and W. Unger, Phase boundary for the chiral transition in $(2+1)$-flavor QCD at small values of the chemical potential, Phys. Rev. D 83, 014504 (2011); R. Falcone, E. Laermann, and M. P. Lombardo, Study of finite temperature QCD with $(2+1)$ flavors via Taylor expansion and imaginary chemical potential, Proc. Sci., LATTICE2010 (2010) 183 [arXiv:1012.4694]; G. Endrodi, Z. Fodor, S. D. Katz, and K. K. Szabo, The QCD phase diagram at nonzero quark density, J. High Energy Phys. 04 (2011) 001; C. Bonati, M. D'Elia, M. Mariti, M. Mesiti, F. Negro, and 
F. Sanfilippo, Curvature of the chiral pseudocritical line in QCD, Phys. Rev. D 90, 114025 (2014); L. Cosmai, P. Cea, and A. Papa, Proc. Sci., LATTICE2014 (2014) 171 [arXiv: 1410.2471].

[11] D. T. Son and M. A. Stephanov, Real time pion propagation in finite temperature QCD, Phys. Rev. D 66, 076011 (2002).

[12] S. Fayazbakhsh, S. Sadeghian, and N. Sadooghi, Properties of neutral mesons in a hot and magnetized quark matter, Phys. Rev. D 86, 085042 (2012); M. Ishii, T. Sasaki, K. Kashiwa, H. Kouno, and M. Yahiro, Effective model approach to meson screening masses at finite temperature, Phys. Rev. D 89, 071901 (2014).

[13] J. I. Kapusta, Finite-Temperature Field Theory (Cambridge University Press, Cambridge, 1989).
[14] B. B. Brandt, A. Francis, H. B. Meyer, and D. Robaina, Chiral dynamics in the low-temperature phase of QCD, Phys. Rev. D 90, 054509 (2014).

[15] Y. Aoki, Z. Fodor, S. D. Katz, and K. K. Szabo, The QCD transition temperature: Results with physical masses in the continuum limit, Phys. Lett. B 643, 46 (2006).

[16] B. B. Brandt, A. Francis, H. B. Meyer, O. Philipsen, and H. Wittig, QCD thermodynamics with $\mathrm{O}(\mathrm{a})$ improved Wilson fermions at $N_{f}=2$, Proc. Sci., LATTICE2013 (2014) 162 [arXiv:1310.8326].

[17] T. Inagaki, D. Kimura, H. Kohyama, and A. Kvinikhidze, Phase diagram of Nambu-Jona-Lasinio model with dimensional regularization, Phys. Rev. D 86, 116013 (2012). 